\title{
Fertility Desires and Intentions and the Relationship to Consistent Condom Use and Provider Communication regarding Childbearing among HIV Clients in Uganda
}

\author{
Glenn J. Wagner, ${ }^{1}$ and Rhoda Wanyenze ${ }^{2}$ \\ ${ }^{1}$ Health Department, RAND Corporation, 1776 Main Street, Santa Monica, CA 90407, USA \\ ${ }^{2}$ School of Public Health, Makerere University, Kampala, Uganda \\ Correspondence should be addressed to Glenn J. Wagner; gwagner@rand.org
}

Received 22 May 2012; Accepted 25 June 2012

Academic Editors: B. Best, R. Bologna, and J. Ogwal-Okeng

Copyright (c) 2013 G. J. Wagner and R. Wanyenze. This is an open access article distributed under the Creative Commons Attribution License, which permits unrestricted use, distribution, and reproduction in any medium, provided the original work is properly cited.

\begin{abstract}
Family planning services emphasize prevention of unplanned pregnancies, but rarely account for the childbearing desires of HIV clients. We examined the correlates of fertility desires and intentions among $767 \mathrm{HIV}$ clients (34\% male) starting antiretrovirals in Uganda. Half of participants had a primary sex partner. Among those with a desire (31\%) or intention (24\%) for having a child in the near future, $60 \%$ had not discussed this with providers. Over one quarter (27\%) were told by their provider that they should not bear a child because of their HIV status. In regression analysis, male gender, younger age, higher CD4, having fewer children, and having a primary partner were associated with fertility desires and intentions; having been told by provider not to have a child was associated with intentions but not desires. Among participants with a primary partner, consistent condom use was greater among those with no fertility intentions, as was receipt of advise about family planning, while HIV disclosure to partner was greater among those with intentions. Partner HIV status was not associated with fertility desires or intentions. These findings highlight the need for reproductive health programs for HIV clients to incorporate safer conception counseling and improve communication regarding childbearing.
\end{abstract}

\section{Background}

Pregnancy in persons living with HIV/AIDS (PLHIV) involves significant public health risks including risks of HIV transmission to uninfected partners (30-40\% of HIVaffected couples are serodiscordant) [1] and the fetus. While prophylactic antiretroviral therapy can prevent mother-tochild transmission [2], over half of the pregnants PLHIV in sub-Saharan Africa do not use such treatment [3]. About $20-40 \%$ of HIV-infected women become pregnant after HIV diagnosis in sub-Saharan Africa [4, 5], and a study in Uganda found that $43 \%$ of pregnancies among HIV-infected women were unplanned [6]. Accordingly, reproductive health programs emphasize preventing pregnancies and use of contraceptives, including consistent condom use [7]. However, this approach does not account for the fact that $20-50 \%$ of PLHIV in sub-Saharan Africa want to have children [1, 4, 8-12].
Childbearing desires may at least partially explain why many PLHIV do not consistently use condoms [13,14], even in the context of serodiscordant relationships where there is a clear risk of horizontal transmission.

To reduce horizontal and vertical transmission, PLHIV must be fully informed about their reproductive options and receive appropriate guidance for safer conception when wanting to conceive. Unfortunately, HIV care and reproductive health services rarely incorporate counseling and services aimed at promoting safer conception. Providers are a critical source of information and counseling regarding reproductive health options for PLHIV, yet providers have been known to convey messages to PLHIV that childbearing is inappropriate and typically do not discuss these issues with clients $[4,15]$. Furthermore, perceived stigma from health care workers and community members may inhibit PLHIV's willingness to initiate conversations about childbearing [4, 15-17]. A study 
of PLHIV in South Africa found that $45 \%$ of women and $57 \%$ of men wanted to have children, yet only $19 \%$ of female and $6 \%$ of male respondents had discussed childbearing options with their HIV provider; most indicated they would welcome such a discussion if given the opportunity [4]. A study in Uganda found that $44 \%$ of HIV clients had fertility intentions, of whom $35 \%$ found it difficult to discuss these intentions with their HIV care providers [18].

Perceived stigma from and inability to communicate with providers about fertility desires may result in a breach of patient trust in providers that could affect reproductive health decisions, access to, and use of reproductive health services including both pre- and postconception risk reduction methods. While preconception risk reduction methods such as sperm washing [19] and artificial insemination are cost-prohibitive [20], ART, timed intercourse, and manual insemination (when the man is HIV negative) are all feasible strategies that can be used to reduce transmission risks by PLHIV who want to conceive [20-22].

We conducted a cross-sectional analysis of the correlates of fertility desires and intentions among HIV clients in Uganda, including those in relationships. We examined whether clients with a primary sex partner who had intentions to bear children were less likely to use condoms consistently, and whether fertility intentions and condom use were associated with relationship factors such as HIV disclosure to the partner, the partner's HIV status, and communication between the client and providers regarding family planning and childbearing.

\section{Methods}

2.1. Study Setting and Sample. Analyses were conducted on data from a longitudinal prospective cohort study of the impact of depression treatment on social and economic outcomes among patients on ART. The study was conducted at four clinics operated by Mildmay Uganda in Kampala, Nagalama, Mukono, and Mityana between September 2010 and February 2012. All clients of age 18 or older who were about to start ART were eligible to participate. Depression was not part of the eligibility criteria, but it was assessed and those who were depressed were treated with antidepressants. Patients were consecutively enrolled as they became eligible, and study interviewers reported that nearly all eligible patients agreed to participate. Although participants were followed for 12 months, data for this analysis come from the baseline assessment only, at which point all participants were about to start ART. Participants were paid 10,000 Uganda shillings to complete the interview, which is consistent with other survey research conducted in the country and is considered an appropriate amount by the Uganda National Council for Science and Technology (UNCST). The study protocol was approved by the Makerere University Research and Ethics Committee, as well as the UNCST.

2.2. Data Collection. All questionnaires were translated into Luganda, which is the primary local language in the study setting, using standard translation and back-translation methods. Questionnaires were interviewer-administered in either Luganda or English, as preferred by the respondent.

2.2.1. Fertility Desires and Intentions. This section of the survey started with the following preamble intended to normalize fertility intentions and diminish stigma-related socially desirable responses: "We know that having children is something that many people think about and value, and that this is no different for people living with HIV. We also know that among people living with HIV there is a wide range of attitudes about child bearing, all of which can be valid and appropriate." To assess fertility desires, respondents were than asked, "Would you like to have (more) children?", if the respondent was in a relationship they were also asked, "Does your partner/spouse want to have (more) children with you?" To assess fertility intentions we asked, "Are you currently planning to have (more) children in the near future?" Response options for the above questions were "Yes" and "No."

\subsubsection{Patient-Provider Communication regarding Childbear-} ing and Family Planning. A number of questions were asked regarding communication between respondents and providers about family planning and childbearing. Participants who reported either wanting or planning to have children in the above questions were asked, "Have you talked to your provider, nurse or counselor about your desire to have a child?" Possible responses to this question consisted of "Yes, with no difficulty," "Yes, with some difficulty," and "No." To assess exposure to family planning counseling, all participants were asked, "Has anyone at this clinic ever spoken to you or asked you about family planning or using contraception to decide when and if you have children?" To assess provider conveyed attitudes about childbearing, respondents were asked, "Have you ever been told by a health care worker that you or your partner/spouse should not become pregnant because of your HIV status?"

2.2.3. Background and Demographic Characteristics. These included age, gender, and education level. Latest CD4 count at time of assessment was abstracted from the patient's clinic chart.

2.2.4. Relationship Characteristics. To assess relationship status, participants were asked if they were married, in a committed relationship, or otherwise had a regular sexual partner. To assess HIV disclosure to the partner and serostatus of the partner, participants who were in a relationship or had a regular sex partner were asked if they had informed their partner of their HIV status, as well as their knowledge of the HIV status of the partner. Condom use during sexual intercourse with one's primary partner and other partners over the past 6 months, in separate items, was assessed using a 5-point rating scale from "never" to "always"; a dichotomous variable was then created to represent whether or not condoms were always used. Number of children that the patient had parented was assessed, and whether or not 
the respondent or their partner was currently pregnant or had given birth to a child within the past 12 months.

2.3. Data Analysis. Descriptive statistics were used to examine the distributions and frequencies of sample characteristics and the primary outcome variables. Bivariate statistics (independent 2-tailed $t$-tests, chi-square tests, Fisher's exact tests) were used to examine whether demographics, relationship characteristics (HIV disclosure, HIV status of partner), condom use with primary partner, and aspects of patientprovider communication regarding childbearing were correlates of fertility desires and intentions. The relationship between fertility intentions and condom use was assessed only within the subgroup of participants with a primary sex partner, not within the whole sample; this is because condom use was assessed mostly among those with a primary sex partner. Condom use with other partners was assessed, but only $7.9 \%$ reported such sex partners, resulting in $56.5 \%$ of the sample having any data on condom use (of whom $89.1 \%$ are participants with a primary partner). Multivariate logistic regression analysis was then performed to predict binary indicators of the presence of fertility desires and intentions.

\section{Results}

3.1. Sample Characteristics. A sample of 767 patients was enrolled in the study and completed the questionnaire related to fertility ( 7 other patients were enrolled but did not complete this section of the survey). The largest contingent of participants was from the Kampala site $(n=338)$, which is the only urban site in the study, followed by Mityana $(n=$ $220)$, Nagalama $(n=117)$, and Mukono $(n=92)$. One-third $(34.0 \%)$ of the sample was male, average age was 36.0 years $(\mathrm{SD}=9.4)$, and $15 \%$ had at least some secondary education. Average time since HIV diagnosis was 24.2 months (median $=14.3$ months; $76 \%$ were diagnosed more than 6 months prior to study enrollment) and mean CD4 cell count was 156 cells $/ \mathrm{mm}^{3}$ (SD $=86$; range: $\left.1-512\right)$.

Nearly one-third $(n=245 ; 31.9 \%)$ were married, and another $12.4 \%(n=96)$ were in a committed relationship, plus 45 other participants reporting having a regular sex partner; hence, a total of $386(50.3 \%)$ had a primary sex partner. Ninety percent had parented at least one child in their lifetime, including $76(10.0 \%)$ who had given birth to a child within the past 12 months; 56 (7.3\%) reported that they or their partners were currently pregnant.

3.2. Prevalence and Correlates of Fertility Desires and Intentions. Thirty-one percent (30.9\%) expressed a desire to have more children, and $23.8 \%$ reported having plans to have a child in the near future. Among those with a desire or plans for having a child, $60.4 \%$ had not discussed this with any of their providers, and most (72.0\%) of those who had told their providers expressed having difficulty doing so. A majority (61.0\%) indicated that someone at the clinic had talked to them about family planning and use of contraception, and $27.4 \%$ reported that a provider had told them that they should not bear a child because of their HIV status.
Table 1 lists results of bivariate analysis examining correlates of fertility desires and intention, in the whole sample. Male gender, younger age, having at least some secondary education, being married or in a committed relationship, having a primary sex partner, having parented fewer children, and having received advise on family planning and contraception use were all positively correlated with both a desire to have more children and having plans to bear a child in the near future. Being told by a provider not to bear children because of their HIV status was not associated with fertility desires, but was associated with a greater likelihood of having an intention to bear a child in the near future. Also, those with fertility intentions had a higher CD4 count. Participants at the three rural sites (Nagalama, Mukono, and Mityana) did not differ significantly from each other on fertility desires (ranged from $22.7 \%$ to $31.5 \%$ ) or fertility intentions (ranged from $17.1 \%$ to $21.7 \%$ ); however, with the rural sites combined, those with fertility desires and intentions were less likely to be from the rural sites compared to the urban Kampala site (see Table 1).

All variables in the bivariate analysis were included in multivariate models to further explore associations; the exception is the binary variable indicating whether or not the respondent is married or in a committed relationship, which we opted to exclude because it overlaps with the variable indicating whether or not the respondent has a primary sex partner. In regression analysis with fertility desires as the independent variable, male gender, younger age, higher CD4, having fewer children, and having a primary partner were all significantly associated with having a desire for more children (see Table 2). In the analysis for fertility intentions, the same five variables that were associated with desires were significantly associated with intentions to conceive a child in the near future, plus having been told by one's provider not to have a child because of HIV status was also more common among those with fertility intentions (see Table 2). Among those with fertility intentions or desires, those who had told their provider about such desires were more likely to have been told by their provider not to have a child because of their HIV status compared to those who had not discussed their childbearing desires with their provider (38.7\% versus $18.3 \%$; chi-square $=12.0, P<.001)$.

\subsection{Fertility Desires and Intentions among Participants with a} Primary Partner and the Association with Relationship Characteristics, Provider Communications Related to Childbearing, and Condom Use. Although $24.1 \%$ and $17.8 \%$ of those who were single or had no regular sex partner reported having fertility desires and intentions, respectively, a significantly greater proportion of those in a relationship or with a primary sex partner had such desires $(37.6 \% ; P<.001)$ and intentions $(29.8 \% ; P<.001)$. Of the 159 participants with a primary partner who had a desire or intention to have children, $61.1 \%$ had not informed their HIV care provider(s) of these desires.

3.3.1. Relationship to HIV Disclosure to Partner and Partner's HIV Status. We explored how fertility desires and intentions were associated with aspects of the participant's relationship 
TABLE 1: Bivariate correlates of fertility desires and intentions.

\begin{tabular}{|c|c|c|c|c|}
\hline & $\begin{array}{l}\text { Fertility desires } \\
\quad(N=530)\end{array}$ & $\begin{array}{l}\text { No fertility desires } \\
\quad(N=237)\end{array}$ & $\begin{array}{l}\text { Fertility intentions } \\
\qquad(N=183)\end{array}$ & $\begin{array}{l}\text { No fertility intentions } \\
\qquad(N=585)\end{array}$ \\
\hline Rural location & $47.7 \%^{* *}$ & $59.6 \%$ & $44.8 \%^{* * *}$ & $59.4 \%$ \\
\hline Male & $44.7 \%{ }^{* * *}$ & $29.2 \%$ & $45.4 \%{ }^{* * *}$ & $30.4 \%$ \\
\hline Mean age & $31.5^{* * *}$ & 38.0 & $31.3^{* * *}$ & 37.5 \\
\hline At least some secondary education & $20.2 \%^{*}$ & $12.8 \%$ & $20.6 \%{ }^{*}$ & $13.4 \%$ \\
\hline Mean CD4 count & 165 & 151 & $169^{*}$ & 151 \\
\hline Married or in relationship & $55.3 \%^{* * *}$ & $39.6 \%$ & $57.4 \%^{* * *}$ & $40.3 \%$ \\
\hline Has a primary sex partner & $61.2 \%{ }^{* * *}$ & $45.5 \%$ & $62.8 \%^{* * *}$ & $46.3 \%$ \\
\hline Mean number of children & $2.4^{* * *}$ & 4.4 & $2.4^{* * *}$ & 4.3 \\
\hline $\begin{array}{l}\text { Providers discussed family planning and } \\
\text { contraception }\end{array}$ & $55.1 \%^{*}$ & $64.2 \%$ & $48.1 \%^{* * *}$ & $65.6 \%{ }^{* * *}$ \\
\hline $\begin{array}{l}\text { Provider said HIV+ patients should not bear } \\
\text { children }\end{array}$ & $26.2 \%$ & $28.3 \%$ & $35.5 \%{ }^{* *}$ & $25.1 \%^{* *}$ \\
\hline
\end{tabular}

${ }^{*} P<.05 ;{ }^{* *} P<.01 ;{ }^{* * *} P<.001$.

* Signifies that the variable differs significantly between those with and without fertility desires, or between those with and without fertility intentions.

with their primary sex partner, including HIV disclosure and HIV status of the partner. Of the 386 participants with a primary partner, $50.4 \%$ reported having a partner who was also HIV-positive, $18.7 \%$ had an HIV-negative partner, and $30.9 \%$ did not know their partner's HIV status. The vast majority $(85.6 \%)$ of participants had disclosed their HIV status to their partner, including nearly all $(98.8 \%)$ of those who knew their partner's HIV status (suggesting the partner had been tested, making it easier to disclose, or that the disclosure led to the partner being tested) compared to just over half $(55.8 \%)$ of those who were unaware of their partner's HIV status. Disclosure of HIV status to one's primary partner was not associated with fertility desires, but fertility intentions were more common among those who had disclosed (32.7\%) compared to those who had not disclosed $(17.0 \%$; F.E.T. $=.02)$. Neither fertility desires nor intentions were associated with HIV status of the partner, with $35 \%$ to $40 \%$ having fertility desires and $25 \%$ to $33 \%$ having intentions across partnerships that were serodiscordant, concordant and where the HIV status of the partner was unknown.

3.3.2. Relationship to Provider Communications regarding Childbearing. Among participants with primary partners, $53.6 \%$ reported that the clinic providers had discussed family planning and contraception use with them, and $32.1 \%$ had been told by a provider that they should not conceive a child because of their HIV status. The proportion of patients whose providers had discussed family planning and contraception use did not differ between those with (53.1\%) and without fertility desires (54.2\%), but fewer participants with fertility intentions received a discussion about family planning (46.1\%) compared to those with no intentions (57.0\%; chi-square $=3.9, P<.05)$. The proportion of patients whose provider expressed a belief that the patient should not conceive a child because of their HIV status did not differ between those with (29.0\%) or without (34.0\%) fertility desires, nor between those with (34.8\%) and without (31.0\%) fertility intentions.
3.3.3. Relationship to Condom Use with Primary Partner. When asked how often condoms were used during sexual intercourse with their primary partner over the past 6 months, $28.1 \%$ reported to "never" use condoms and $44.0 \%$ said they "always" used condoms, with the remaining 27.9\% reporting use of condoms either "rarely," "sometimes," or "often." Consistent condom use (i.e., always using condoms) over the past 6 months was greater among those without fertility desires $(48.2 \%$ versus $37.0 \%$; chi-square $=4.4 ; P<$ $.05)$ and without fertility intentions (36.6\% versus $29.8 \%$; chisquare $=7.9 ; P<.01)$ compared to those with fertility desires and intentions. Among patients who reported no intentions of having children in the near future, their partner's desire for childbearing was marginally associated with condom use, as consistent condom use was greater among those whose partner did not want a child compared to those whose partner did want a child $(51.7 \%$ versus $37.7 \% ; P<.10)$.

\section{Discussion and Conclusions}

Our study findings add further evidence of how common childbearing desires and intentions are among PLHIV in subSaharan Africa, and not only among those in relationships, and regardless of whether one's partner is HIV-infected or not. We also found that those with fertility desires or intentions are less likely to consistently use condoms during sex with their primary partners, presumably due at least in part to attempts to increase the likelihood of conception. These results highlight the need for HIV care and reproductive health programs to recognize the fertility rights of PLHIV and to begin to incorporate services to support safer conception and childbearing.

Unfortunately, our data indicate that patients do not feel comfortable communicating their fertility desires with providers, effectively preventing an opportunity to learn about options for safer conception and limiting risks of horizontal transmission. 
TABLE 2: Multivariate correlates of fertility desires and intentions.

\begin{tabular}{lcc}
\hline & $\begin{array}{c}\text { Fertility desires O.R. } \\
(95 \% \text { C.I.s })\end{array}$ & $\begin{array}{c}\text { Fertility intentions O.R. } \\
(95 \% \text { C.I.s })\end{array}$ \\
\hline Female gender & $0.34(0.22-0.53)^{* * *}$ & $0.35(0.22-0.56)^{* * *}$ \\
Age & $0.92(0.89-0.95)^{* * *}$ & $0.93(0.90-0.96)^{* * *}$ \\
Any secondary education & $1.40(0.84-2.39)$ & $1.34(0.79-2.30)$ \\
Urban location & $0.90(0.61-1.35)$ & $1.01(0.66-1.55)$ \\
Number of parented children & $0.80(0.72-0.88)^{* * *}$ & $0.82(0.73-0.91)^{* * *}$ \\
CD4 cell count & $1.00(1.00-1.00)^{*}$ & $1.00(1.00-1.01)^{*}$ \\
Has a primary sex partner & $2.10(1.40-3.15)^{* * *}$ & $2.10(1.35-3.26)^{* *}$ \\
Provider(s) discussed family planning and contraception use & $0.88(0.58-1.34)$ & $0.74(0.47-1.15)$ \\
Provider advised against childbearing because of HIV status & $0.82(0.52-1.29)$ & $1.74(1.09-2.78)^{*}$ \\
\hline
\end{tabular}

${ }^{*} P<.05 ;{ }^{* *} P<.01 ;{ }^{* * *} P<.001$.

The rates of participants who expressed a desire to have children $(31 \%)$ and have a plan to bear a child in the near future $(24 \%)$ are similar to what other studies have found in sub-Saharan Africa [1, 4, 8-12]. Furthermore, nearly one in five participants reported either parenting in the past year or about to parent a child. There is no disput that childbearing is as important to PLHIV as their noninfected counterparts. African social and cultural values dictate that having children is key to the identity and social status of men and women, including for PLHIV [15, 23, 24]. Childbearing helps secure a marriage, and lineage continuity and family often apply pressure to reproduce [1]. Consistent with other studies, our data found fertility desires and intentions to be positively correlated with male gender, younger age, having fewer children, better health status, and being in a relationship $[4,11,25-27]$.

Our finding that fertility desires were unrelated to the HIV status of the patient's partner, and that intentions to bear children were as high in partnerships where the partner was HIV-negative or of unknown HIV status as they were in seroconcordant partnerships, reveals the risk for horizontal transmission presented by childbearing in this population. This risk is heightened even further by our finding that those with fertility desires or intentions, or if their primary partner has fertility desires even if they don't, are less likely to consistently use condoms during sex with their primary partner. Consistent with other studies of PLHIV in the region [28-30], over half of the participants reported unprotected sexual intercourse with their primary partners, including over two-thirds of those with fertility desires or intentions. Clearly, HIV prevention efforts aimed at promoting condom use among PLHIV must account for the fact that inconsistent condom use is attributed at least in part to desires for childbearing.

Effective safer conception counseling requires feasible conception risk reduction methods. Assisted reproductive techniques such as sperm washing [19] and artificial insemination are clearly cost-prohibitive for the vast majority of PLHIV [20], but low-cost methods for safer conception are also available. Manual insemination with an uninfected male's sperm when the woman is HIV-infected carries no risk for horizontal transmission and bears little cost. The amount of risk reduction associated with timed unprotected sex in discordant couples where the woman is uninfected is uncertain, but logically evident, especially if the infected male is adherent to antiretroviral therapy and any sexually transmitted infections are treated [20-22]. Data from HPTN052 show that risk for infection among serodiscordant couples is reduced by $96 \%$ with the use of ART [31]. Early studies of preexposure prophylaxis (PrEP) with heterosexual serodiscordant couples have been mixed [32-34], but this may also prove to be an option for promoting safe conception. Little is known about how aware PLHIV are of these safer conception methods or how willing they are to use them. Clinicians may not even have a clear understanding of these methods. Clearly this is an area of need for future research programmatic efforts aimed at incorporating safer counseling services into reproductive health care.

With the need for safer conception counseling being apparent, it is troubling how few of the participants with fertility desires or intentions in our study had informed their providers. Furthermore, most of those who had discussed their desires and intentions with their provider indicated that telling their provider was difficult or uncomfortable. Other studies have found similar evidence of poor communication between providers and patients regarding childbearing desires [4], including our own research in Uganda [18]. Providers have been known to convey messages to PLHIV that childbearing is inappropriate $[4,15,16]$, and one-quarter of our study participants reported receiving such sentiments from their providers. In fact, our study participants who did tell their provider(s) about their desires to have a child were more likely to have been told by their providers that they should not have a child because of their HIV status, and probably did not receive the support they were seeking for their childbearing needs. Perceived stigma from and inability to communicate with providers about fertility desires may result in a breach of patient trust in providers that could affect reproductive health decisions, as well as engagement in and adherence to HIV care, which may have implications for access to and use of reproductive health services, safety of childbearing, and infectiousness.

Limitations of the study include the limited scope of our enquiry regarding communication of fertility intentions with 
providers. As this was not the primary purpose of the larger study, we did not assess why clients had difficulty talking with providers about their childbearing plans, and whether perceived stigma from providers was a contributing factor. Our large sample size improves the generalizability of our findings to other PLHIV receiving free HIV care in similar clinical settings, but not to the overall population of HIVinfected individuals, and in particular those not receiving care or who do not know their HIV status, as well as those who have been on ART. Studies conducted in sub-Saharan Africa suggest that knowledge of one's HIV infection is associated with a reduction in desire and intention to have more children $[35,36]$.

In conclusion, the results of our study reinforce the message that sexual and reproductive health programs can no longer focus almost entirely on prevention of unplanned pregnancies and mother-to-child transmission, but must also acknowledge the needs of PLHIV to have children and thus provide the necessary counseling and services to support clients to conceive and have children safely. It is time for "family planning" to no longer be synonymous with an emphasis on contraception for preventing pregnancy, but to represent the full meaning of its name by also helping clients and couples to achieve their goal of having children and families and to do so as safely and responsibly as possible. Promoting use of safer conception methods in PLHIV could be critical to averting new HIV infections; hence, the need for further research to better understand the factors influencing the use of such methods, and to enhance reproductive service provision within HIV care to assist PLHIV with intentions to have children.

\section{Recommendations}

Policy makers and service providers need to add safer conception counseling and support services to the armament of reproductive health and family planning services for PLHIV. Furthermore, HIV care providers should take a more proactive approach to initiate discussions with clients about fertility desires and intentions, so as to facilitate timely service provision regarding safer conception support services and limiting risk of horizontal and vertical HIV transmission.

\section{Acknowledgment}

This research is funded by a Grant 5R01MH083568 from the National Institute of Mental Health (PI: G. J. Wagner).

\section{References}

[1] J. Beyeza-Kashesya, F. Kaharuza, F. Mirembe, S. Neema, A. M. Ekstrom, and A. Kulane, "The dilemma of safe sex and having children: challenges facing HIV sero-discordant couples in Uganda," African Health Sciences, vol. 9, no. 1, pp. 2-12, 2009.

[2] J. H. Tai, M. A. Udoji, G. Barkanic et al., "Pregnancy and HIV disease progression during the era of highly active antiretroviral therapy," Journal of Infectious Diseases, vol. 196, no. 7, pp. 1044-1052, 2007.
[3] E. M. Stringer, D. K. Ekouevi, D. Coetzee et al., "Coverage of nevirapine-based services to prevent mother-to-child HIV transmission in 4 African countries," Journal of the American Medical Association, vol. 304, no. 3, pp. 293-302, 2010.

[4] D. Cooper, J. Moodley, V. Zweigenthal, L. G. Bekker, I. Shah, and L. Myer, "Fertility intentions and reproductive health care needs of people living with HIV in Cape Town, South Africa: implications for integrating reproductive health and HIV Care services," AIDS and Behavior, vol. 13, supplement 1, pp. S38-S46, 2009.

[5] S. Allen, J. Meinzen-Derr, M. Kautzman et al., "Sexual behavior of HIV discordant couples after HIV counseling and testing," AIDS, vol. 17, no. 5, pp. 733-740, 2003.

[6] R. K. Wanyenze, N. M. Tumwesigye, R. Kindyomunda et al., "Uptake of family planning methods and unplanned pregnancies among HIV-infected individuals: a cross-sectional survey among clients at HIV clinics in Uganda," Journal of the International AIDS Society, vol. 14, no. 1, article 35, 2011.

[7] World Health Organization (WHO), Reproductive Health Strategy to Accelerate Progress Towards the Attainment of International Development Goals and Targets, World Health Organization, Geneva, Switzerland, 2004, http://www.who.int/ reproductive-health/strategy.html/.

[8] A. Kaida, I. Andia, M. Maier et al., "The potential impact of antiretroviral therapy on fertility in sub-Saharan Africa," Current HIV/AIDS Reports, vol. 3, no. 4, pp. 187-194, 2006.

[9] L. Myer, C. Morroni, and K. Rebe, "Prevalence and determinants of fertility intentions of HIV-infected women and men receiving antiretroviral therapy in South Africa," AIDS Patient Care and STDS, vol. 21, no. 4, pp. 278-285, 2007.

[10] B. Nattabi, J. Li, S. C. Thompson, C. G. Orach, and J. Earnest, "A systematic review of factors influencing fertility desires and intentions among people living with HIV/AIDS: implications for policy and service delivery," AIDS and Behavior, vol. 13, no. 5, pp. 949-968, 2009.

[11] M. Maier, I. Andia, N. Emenyonu et al., "Antiretroviral therapy is associated with increased fertility desire, but not pregnancy or live birth, among HIV+ women in an early HIV treatment program in rural Uganda," AIDS and Behavior, vol. 13, supplement 1, pp. S28-S37, 2009.

[12] V. Ndlovu, "Considering childbearing in the age of highly active antiretroviral therapy (HAART): views of HIV-positive couples," Journal of Social Aspects of HIV-AIDS, vol. 6, no. 2, pp. 58-68, 2009.

[13] K. Ngure, N. Mugo, and C. Celum, "A qualitative study of barriers to consistent condom use among HIV-1 serodiscordant couples in Kenya," AIDS Care, vol. 24, no. 4, pp. 509-516, 2011.

[14] R. Bunnell, A. Opio, J. Musinguzi et al., "HIV transmission risk behavior among HIV-infected adults in Uganda: results of a nationally representative survey," AIDS, vol. 22, no. 5, pp. 617-624, 2008.

[15] V. Agadjanian and S. R. Hayford, "PMTCT, HAART, and childbearing in Mozambique: an institutional perspective," AIDS and Behavior, vol. 13, no. 1, pp. S103-S112, 2009.

[16] M. Nduna and L. Farlane, "Women living with HIV in South Africa and their concerns about fertility," AIDS and Behavior, vol. 13, supplement 1, pp. S62-S65, 2009.

[17] L. Myer, C. Morroni, and D. Cooper, "Community attitudes towards sexual activity and childbearing by HIV-positive people in South Africa," AIDS Care, vol. 18, no. 7, pp. 772-776, 2006. 
[18] G. Wagner, S. Linnemayr, C. Kityo, and P. Mugyenyi, "Factors associated with intention to conceive and its communication to providers among HIV clients in Uganda," Maternal and Child Health Journal, vol. 16, no. 2, pp. 510-518, 2012.

[19] A. E. Semprini, A. Vucetich, and L. Hollander, "Sperm washing, use of HAART and role of elective Caesarean section," Current Opinion in Obstetrics and Gynecology, vol. 16, no. 6, pp. 465-470, 2004.

[20] L. T. Matthews and J. S. Mukherjee, "Strategies for harm reduction among HIV-affected couples who want to conceive," AIDS and Behavior, vol. 13, no. 1, pp. S5-S11, 2009.

[21] P. Barreiro, J. del Romero, M. Leal et al., "Natural pregnancies in HIV-serodiscordant couples receiving successful antiretroviral therapy," Journal of Acquired Immune Deficiency Syndromes, vol. 43, no. 3, pp. 324-326, 2006.

[22] P. Barreiro, J. A. Castilla, P. Labarga, and V. Soriano, "Is natural conception a valid option for HIV-serodiscordant couples?" Human Reproduction, vol. 22, no. 9, pp. 2353-2358, 2007.

[23] E. Preston-whyte, "Culture, context and behaviour: anthropological perspectives on fertility in Southern Africa," Southern African Journal of Demography, vol. 2, no. 1, pp. 13-23, 1988.

[24] S. Sonko, "Fertility and culture in sub-Saharan Africa: a review," International Social Science Journal, vol. 46, no. 3, pp. 397-411, 1994.

[25] J. Beyeza-Kashesya, A. M. Ekstrom, F. Kaharuza, F. Mirembe, S. Neema, and A. Kulane, "My partner wants a child: a crosssectional study of the determinants of the desire for children among mutually disclosed sero-discordant couples receiving care in Uganda," BMC Public Health, vol. 10, article 247, 2010.

[26] A. A. Nóbrega, F. A. S. Oliveira, M. T. G. Galvão et al., "Desire for a child among women living with HIV/AIDS in northeast Brazil," AIDS Patient Care and STDs, vol. 21, no. 4, pp. 261-267, 2007.

[27] O. T. Oladapo, O. J. Daniel, O. L. Odusoga, and O. AyoolaSotubo, "Fertility desires and intentions of HIV-positive patients at a suburban specialist center," Journal of the National Medical Association, vol. 97, no. 12, pp. 1672-1681, 2005.

[28] T. P. Eisele, C. Mathews, M. Chopra et al., "Changes in risk behavior among HIV-positive patients during their first year of antiretroviral therapy in Cape Town South Africa," AIDS and Behavior, vol. 13, no. 6, pp. 1097-1105, 2009.

[29] B. O. Olley, S. Seedat, F. Gxamza, H. Reuter, and D. J. Stein, "Determinants of unprotected sex among HIV-positive patients in South Africa," AIDS Care, vol. 17, no. 1, pp. 1-9, 2005.

[30] G. J. Wagner, I. Holloway, B. Ghosh-Dastidar, G. Ryan, C. Kityo, and P. Mugyenyi, "Factors associated with condom use among HIV clients in stable relationships with partners at varying risk for HIV in Uganda," AIDS and behavior, vol. 14, no. 5, pp. 1055-1065, 2010.

[31] The Lancet, "HIV treatment as prevention-it works," The Lancet, vol. 377, no. 9779, p. 1719, 2011.

[32] Family Health International (FHI), "FEM-PrEP June 2011," http://www.fhi360.org/en/AboutFHI/Media/Releases/FEMPrEP_statement041811.htm.

[33] Partners PrEP Study, "Pivotal study finds that HIV medications are highly effective as prophylaxis against HIV infection in men and women in Africa. Press Release," 2011, http:// depts.washington.edu/uwicrc/research/studies/PrEP.html.

[34] P. L. Vernazza, I. Graf, U. Sonnenberg-Schwan, M. Geit, and A. Meurer, "Pre-exposure prophylaxis and timed intercourse for HIV-discordant couples willing to conceive a child," AIDS, vol. 25, no. 16, pp. 2005-2008, 2011.
[35] J. Heys, W. Kipp, G. S. Jhangri, A. Alibhai, and T. Rubaale, "Fertility desires and infection with the HIV: results from a survey in rural Uganda," AIDS, vol. 23, no. 1, pp. S37-S45, 2009.

[36] F. Taulo, M. Berry, A. Tsui et al., "Fertility intentions of HIV1 infected and uninfected women in Malawi: a longitudinal study," AIDS and Behavior, vol. 13, supplement 1, pp. S20-S27, 2009. 


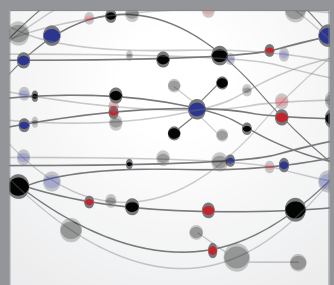

The Scientific World Journal
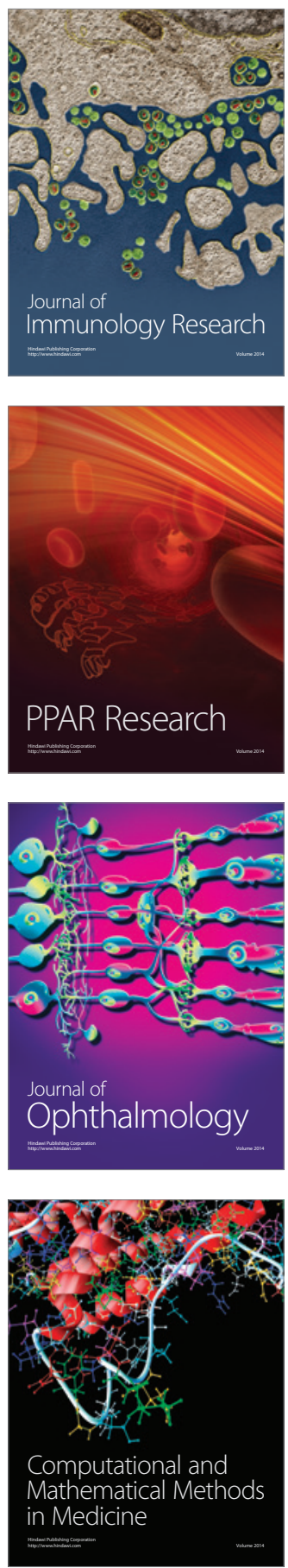

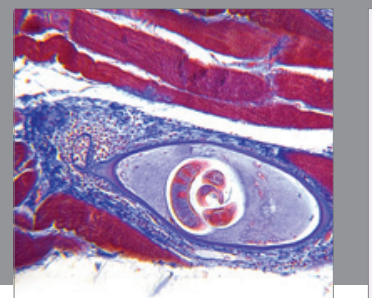

Gastroenterology

Research and Practice
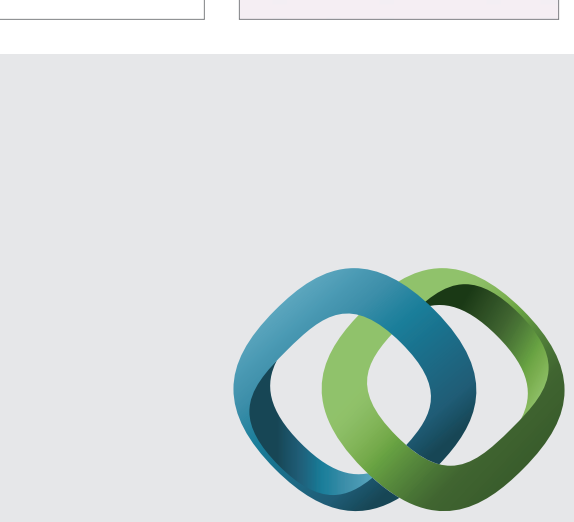

\section{Hindawi}

Submit your manuscripts at

http://www.hindawi.com
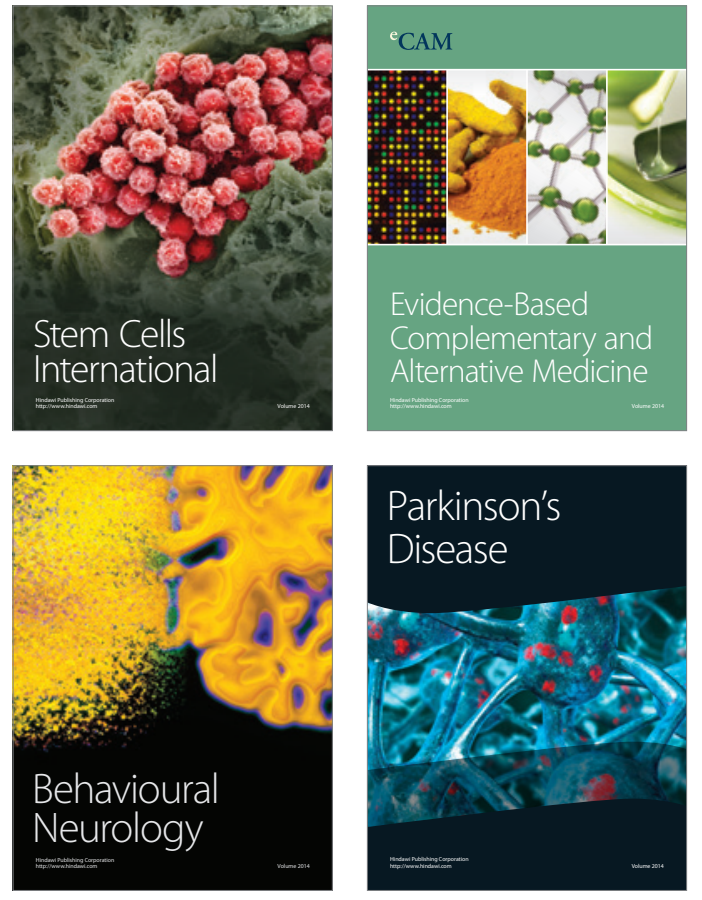
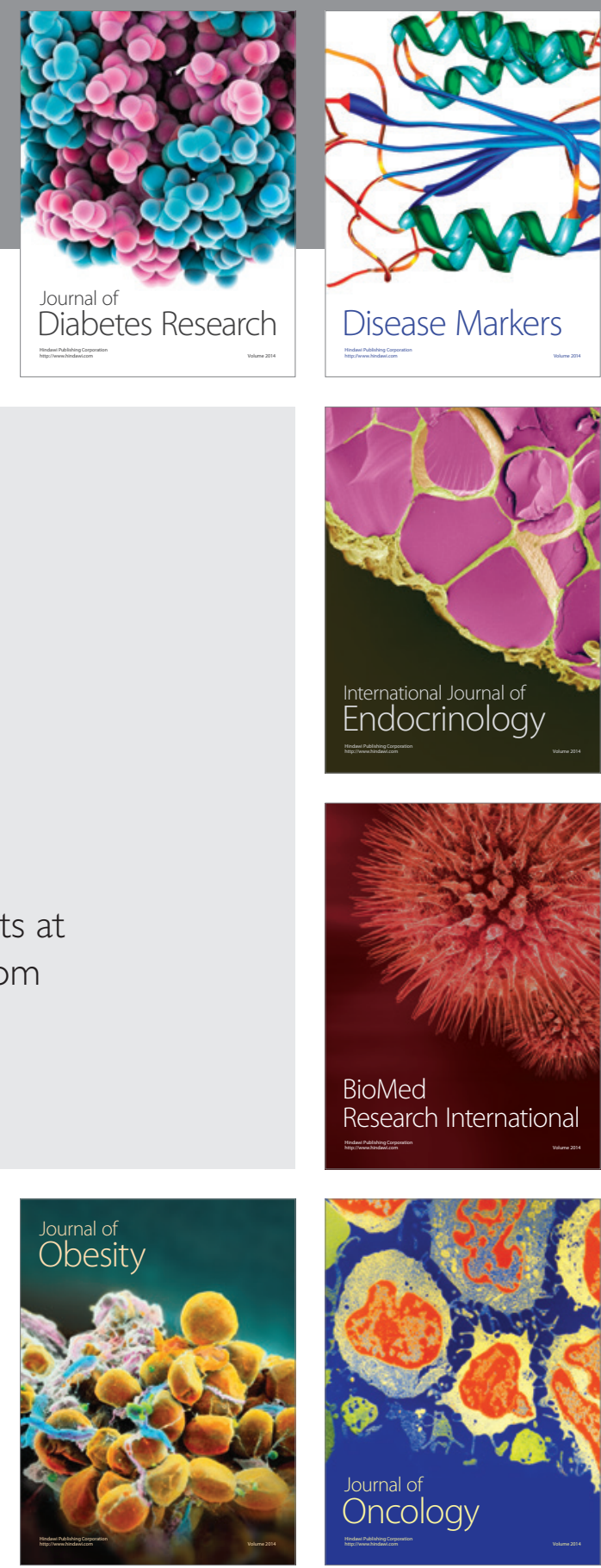

Disease Markers
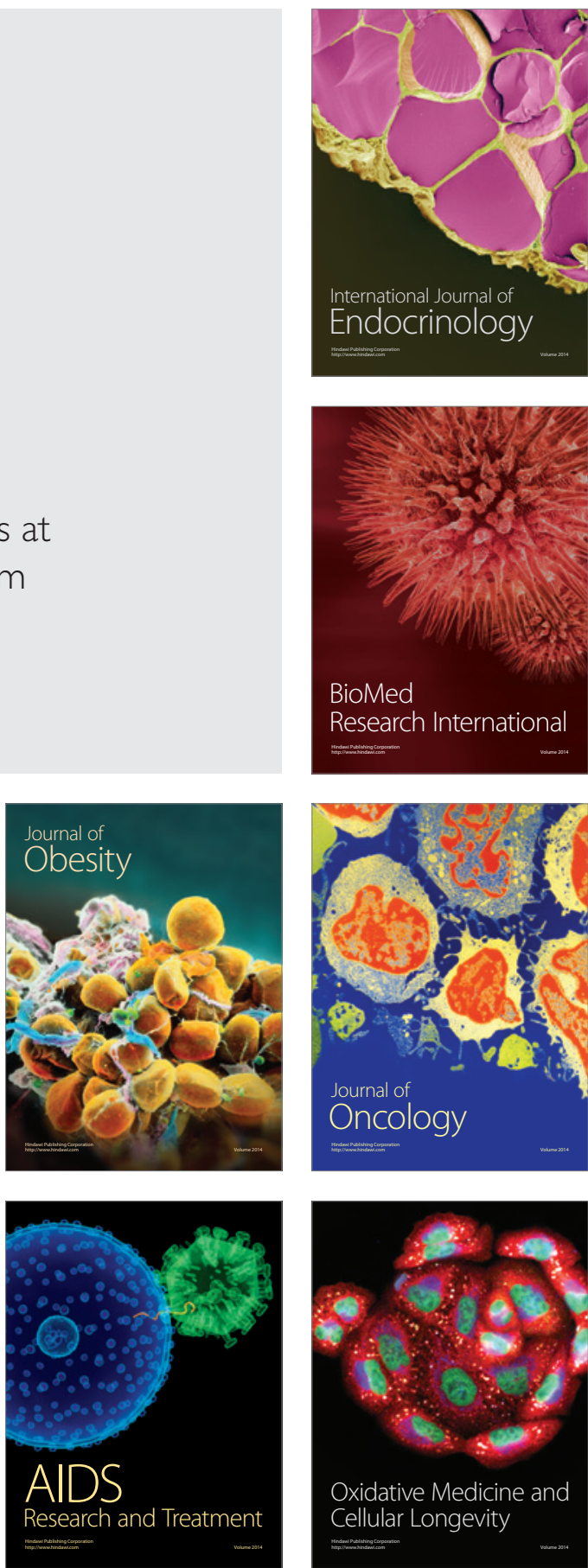